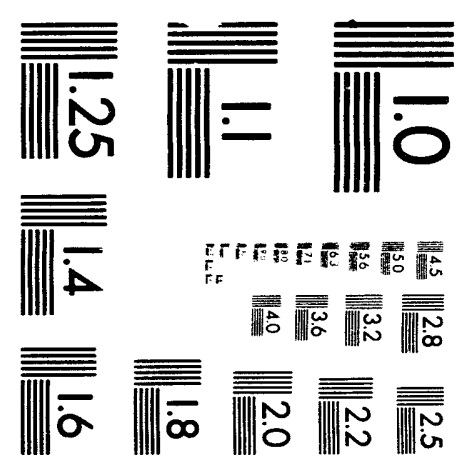



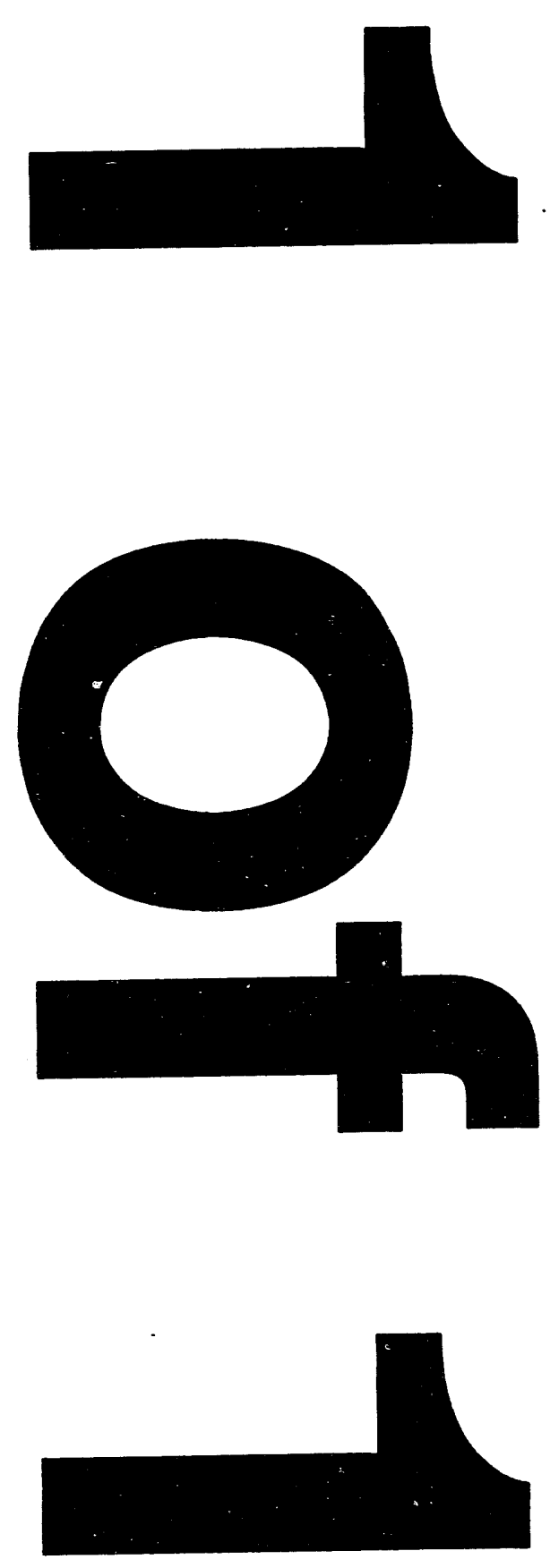
Modeling Turbulence in Flows with a Strong Rotational Component

Don E. Burgess

Peter J. O'Rourke 


\title{
MODELING TURBULENCE IN FLOWS WITH A STRONG ROTATIONAL COMPONENT
}

by

Don E. Burgess and Peter J. O'Rourke

\begin{abstract}
We consider the effectiveness of various turbulence models in flows with a strong rotational component. To evaluate the models, we implement them into a one-dimensional test code and make comparisons with experimental data for swirling flow in a cylinder. ${ }^{1}$ The $K-\varepsilon$ type turbulence models do poorly in predicting the experimental results. However, we find that the incorporation of a Reynolds stress evolution equation gives good agreement with the experimentally measured mean flow. Modeling the pressure-strain correlation tensor correctly is the key for obtaining good results. A combination of Launder's basic modei ${ }^{2}$ together with Yakhot's dissipation rate equation ${ }^{3}$ works best in predicting both the mean flow and the turbulence intensity.
\end{abstract}

\section{Introduction}

We are interested in finding a turbulence model that can predict turt $v^{\prime} \cdot$ ice $^{-}$; ransport in the presence of strong swirl. The primary motivation is to develop a bet wa turbulence model that can be used in the simulation of internal combustion engines. In addition, our studies may be relevant to geophysical flows, which usually involve rotational aff cts. Significant vorticity is introduced into internal combustion engines to promote com', wion by creating swirl-induced shears. Hence it is crucial to understand the interactio : tetween turbulence and rotational effects in order to accurately design internal combustion engines. Unfortunately, the standard turbulence models now in use break down in the presence of streamline curvature. 2,4

Wahiduzzaman and Ferguson ${ }^{1}$ did an experiment at Purdue in a simple cylindrical geometry which combined buoyancy and rotational effects on turbulence. Throughout this paper, we will refer to this experiment as the "Purdue experiment". When we incorporate various models into a one-dimensional, incompressible test code, we will make comparisons between computed results 
and the experimental data from the Purdue experiment. Though the experimental geometry is simple, it has been surprisingly difficult to model the turbulent transport of momentum and heat in this experiment.

Initially, Wahiduzzaman and Ferguson ${ }^{1}$ evacuated a cylindrical vessel. Then, they introduced heated Argon tangentially into the vessel. As the Argon gas entered the vessel, it expanded into the low-pressure environment and absorbed heat from the walls. As the gas filled the vessel, new gas entering the vessel underwent less expansion as the pressure inside the vessel increased and, so, absorbed less heat from the walls. Consequently, the gas introduced first was the most. energetic; and as it was adiabatically compressed towards the center of the cylinder, it developed a higher temperature than the surrounding gas. In this way, Wahiduzzaman and Ferguson obtained a swirling flow with a high-temperature region in the center of the cylinder. Subsequently, they made an ensemble average of their data to obtain the mean temperature and velocity fields as the swirling flow decayed. In addition, they measured the average heat loss at the top of the cylinder as well as the turbulent kinetic energy.

Even though the standard $K-\varepsilon$ turbulence model is the industrial standard in computing combustion flows, it does poorly in predicting this simple experimental flow. Figure 1 shows a time sequence of azimuthal velocity profiles for calculations done with the $K-\varepsilon$ turbulence model. The computed velocity profiles look much like a solid-body rotation while the experimental profiles are strongly curved.

In this report, we concentrate on rotational effects on incompressible turbulence. Though the Purdue experiment had density variations on the order of $30 \%$, preliminary calculations showed that centrifugal effects due to these density variations had a negligible effect on the mean velocity profiles. Evidently, errors in the calculated velocities were caused by inaccuracies of the basic incompressible $K-$ s model.

In Section II, we discuss the equations of motion for incompressible, constant density turbulence. We show the importance of formulating the equations in covariant form to make frame-independent. modeling assumptions. Then, in Section III, we describe how well various turbulence models predict the mean flow and the turbulence intensity measured in the Purdue experiment. We find that a combination of Launder's basic model $^{2}$ and the RNG $\varepsilon$-equation ${ }^{3}$ works best. Finally, in Section IV, we discuss the results of our calculations and our plans for the future.

\section{Equations of Motion}

\section{A Introduction}

In this section, we discuss the equations of motion for incompressible, constant density turbulence. We consider both eddy-viscosity closures and second moment closures for the Reynolds stress tensor. We conclude this section with a discussion of the turbulent boundary layer treatment.

Conventionally, one expresses the equations of motion in Cartesian coordinates. To transform the equations to a curvilinear coordinate system, one can use the chain rule. Alternatively, one can put the equations in a covariant form. In a general curvilinear coordinate system, the chain rule introduces additional terms because the basis vectors depend on position. In order to make frame independent modeling assumptions, one should put the equations in covariant form to see the additional terms explicitly. So, we will use tensor equations throughout this section. 
Swirl Velocity with K- $\varepsilon$ Model
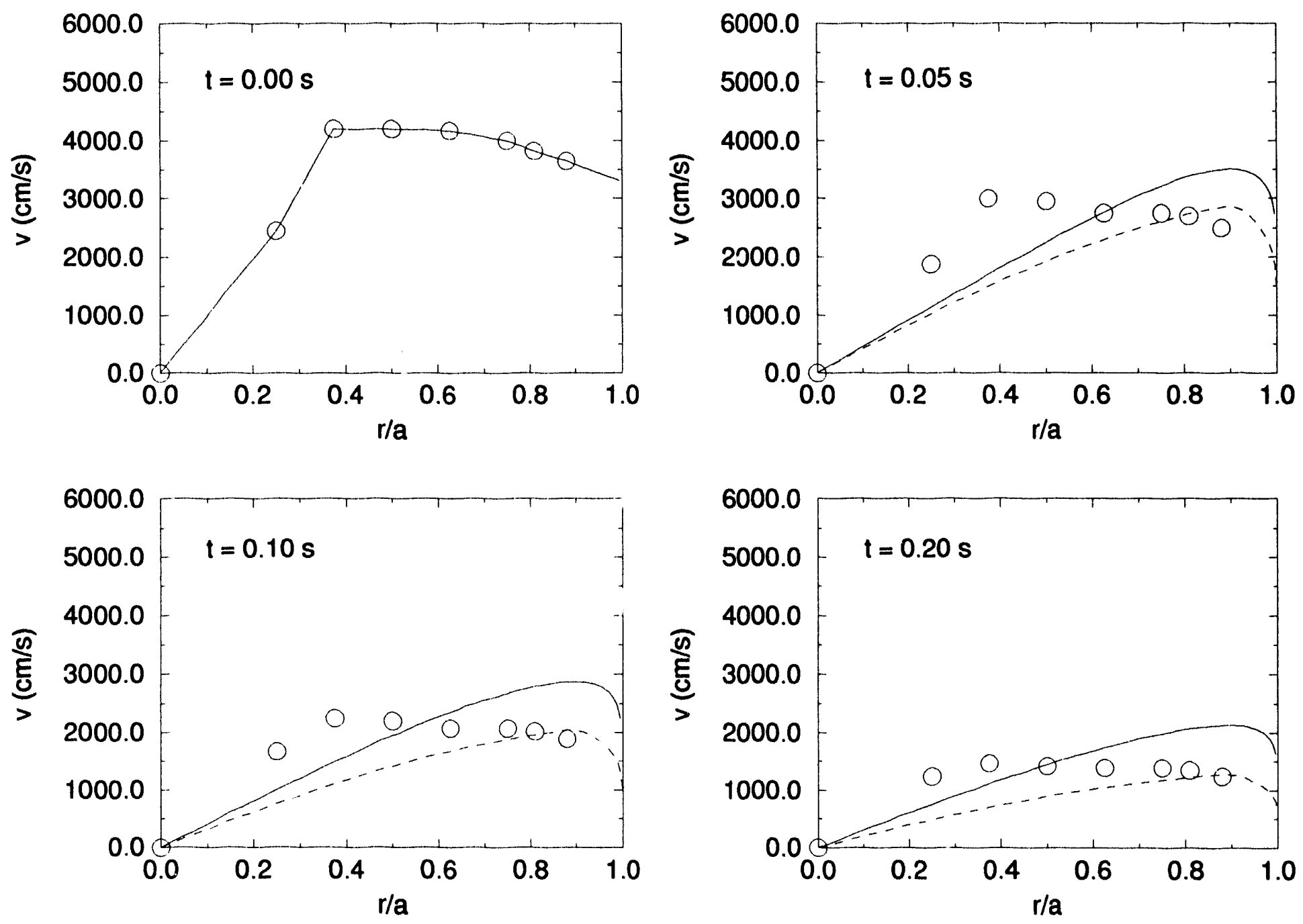

Figure 1: A time sequence of the azimuthal velocity profiles along the central plane as a function of radial position $r$ divided by the cylinder radius $a$. The solid lines are from a one-dimensional calculation, the dashed lines are from a two-dimensional KIVA calculation, and the circles correspond to experimental measurements. Both calculations were done using the standard $K-\varepsilon$ model. 


\section{B Covariant Formulation of the Equations of Motion}

First, we briefly review covariant tensor notation and formulas. For a useful introduction to differential geometry, refer to Schutz. ${ }^{5}$ Letters with superscript indices are vector components, while letters with subscript indices are one-form (covariant vector) components. The metric tensor $g$ maps vector components into one-form components via

$$
v_{i}=g_{i j} v^{j}
$$

Correspondingly, the inverse metric tensor $\boldsymbol{g}^{-1}$ maps one-form components to vector components

$$
v^{i}=g^{i j} v_{j}
$$

This notation is generalized to tensors of arbitrary rank in a natural way.

As is well known, the partial derivative of a tensor with respect to $x^{j}$ is not a tensor. The covariant derivative is the tensor which reduces to partial differentiation in Cartesian coordinates. If $\boldsymbol{T}$ is an $\left(\begin{array}{c}N \\ M\end{array}\right)$ tensor, then the covariant derivative of $\boldsymbol{T}$ is given by

$$
\begin{aligned}
T_{k \ldots l}^{i \ldots j}{ }_{k m} & =T_{k \ldots l}^{i \ldots j}{ }_{k \ldots l} \\
& +\left\{\begin{array}{c}
i \\
n m
\end{array}\right\} T_{k \ldots l}^{n \ldots j}+\ldots+\left\{\begin{array}{c}
j \\
n m
\end{array}\right\} T_{k \ldots l}^{i \ldots n} \\
& -\left\{\begin{array}{c}
n \\
k m
\end{array}\right\} T^{i \ldots \ldots}{ }_{n \ldots l}^{i \ldots j}-\ldots-\left\{\begin{array}{c}
n \\
l m
\end{array}\right\} T_{k \ldots n}^{i \ldots j},
\end{aligned}
$$

where a semicolon followed by subscript $m$ denotes the covariant derivative of $\boldsymbol{T}$ along $x^{m}$, a comma followed by subscript $m$ denotes the usual partial derivative of $T$ with respect to $x^{m}$, and $\left\{\begin{array}{c}s \\ i k\end{array}\right\}$ are Christoffel's symbols. If $z^{k}$ are Cartesian coordinates and $x^{k}$ are some curvilinear coordinates, then the Christoffel's symbols are expressed as

$$
\left\{\begin{array}{c}
s \\
i j
\end{array}\right\}=\frac{\partial^{2} z^{k}}{\partial x^{i} \partial x^{j}} \frac{\partial x^{s}}{\partial z^{k}}
$$

So for example, the convective term $v^{k} v^{i},{ }_{k}$ is not a tensor. To make the convective term a tensor, one can use the covariant derivative $v_{i} ; j$, namely,

$$
v^{k} v_{i ; k}=v^{k} v_{i, k}-\left\{\begin{array}{c}
s \\
i k
\end{array}\right\} v^{k} v_{s}
$$

where we have used the covariant derivative formula Eq. (II.1). The convective tensor $v^{k} v_{i} ;$ is a one-form component.

As an aside, we can write a frame-independent form of the convective term without the use of Christoffels's symbols. The following relationship between the metric tensor and Christoffel's symbols,

$$
g^{i j}, k=-g^{i s}\left\{\begin{array}{c}
j \\
k s
\end{array}\right\}-g^{s j}\left\{\begin{array}{c}
i \\
k s
\end{array}\right\}
$$


just says that the covariant derivative of the metric tensor is zero. (Refer to Eq. (II.1).) Using Eq. (II.2), we can write the convective tensor as

$$
v^{k} v_{i},_{k}=v^{k} v_{i, k}+\frac{1}{2}\left(v_{k} v^{k}{ }_{i}-v^{k} v_{k, i}\right),
$$

which contains no Christoffel's symbols.

This frame-independent group of terms presented in Eq. (II.3) explicitly shows the origin of the so called "centrifugal" and "Coriolis" terms that arise when the convective terms are written in cylindrical coordinates. These terms arise in an inertial frame and are not due to fictitious forces. In an analogous fashion, when the Reynolds stress equations are written in a frame-independent fashion, one can see how the Reynolds stress tensor changes with time in a general curvilinear coordinate system.

\section{Mean Flow Equations}

For incompressible flows, we solve the following system of equations,

$$
\begin{aligned}
\frac{\partial v_{i}}{\partial t}+v^{k} v_{i} ; k & =-\left(\frac{p}{\rho}\right){ }_{i}-R_{i}{ }^{k} ;_{k} \text { and } \\
v^{k} ; k & =0,
\end{aligned}
$$

where $v_{i}$ is the $i$ th component of the mean velocity and $R_{i j}$ is the Reynolds stress tensor, given in terms of the fluctuating velocity components $v_{i}^{\prime}$ by

$$
R_{i j}=\overline{v_{i}^{\prime} v_{j}^{\prime}}
$$

The Reynolds stress tensor, $R_{i j}$, represents the stress that results from the turbulent transport of momentum. The goal of turbulence modeling is to find a closed set of equations for $R_{i j}$.

\section{D $\quad k-\varepsilon$ 'Turbulence Models}

A very popular and simple turbulence closure is the $K-\varepsilon$ model. The standard $K-\varepsilon$ model uses an eddy-viscosity type of closure for $R_{i j}$, namely,

$$
R_{i j}-\frac{2}{3} K \delta_{i j}=-2 \nu_{t} S_{i j}
$$

where the turbulent viscosity $\nu_{t}$ is given in terms of the turbulent kinetic energy $K=\frac{1}{2} R_{k}^{k}$ and the turbulent energy dissipation rate $\varepsilon$ by

$$
\nu_{t}=c_{\mu} \frac{h^{2}}{\varepsilon}
$$

and where the mean symmetric rate of strain tensor $S_{i j}$ is

$$
S_{i j}=\frac{1}{2}\left(v_{i} i_{j}+v_{j i}\right) \text {. }
$$

The $K-\varepsilon$ closure is completed with transport equations for $K$ and $\varepsilon$, namely,

$$
\begin{aligned}
\frac{D K}{D t} & =-R^{i j} S_{i j}-\varepsilon+\left(\frac{\nu_{t}}{\mathrm{Pr}_{K}} H_{k}\right) ; k \text { and } \\
\frac{D E}{D t} & =-c_{\varepsilon 1} \frac{\varepsilon}{h} R^{i j} S_{i j}-c_{\varepsilon} \frac{\varepsilon^{2}}{h}+\left(\frac{\nu_{t}}{\mathrm{Pr}_{\varepsilon}} \varepsilon_{k}\right) ; k,
\end{aligned}
$$


where the nonlinear diffusion terms model the turbulent transport of $K$ and $\varepsilon$.

Using renormalization group analysis (RNG), Yakhot $c t$ al. recently int roduced a generalization of the RNG $K-\varepsilon$ turbulence model. ${ }^{3}$ The $K$-equation is essentially the same with a slightly different value for $\operatorname{Pr}_{K}$. However, in the RNG dissipation rate transport equation, $c_{\varepsilon 1}$ is no longer a constant, but is a function of the dimensionless shear $\eta$, namely,

$$
\frac{D \varepsilon}{D t}=-c_{\varepsilon 1}^{*} \frac{\varepsilon}{h} R^{i j} S_{i j}-c_{\varepsilon 2} \frac{\varepsilon^{2}}{\Lambda^{*}}+\left(\frac{\nu_{t}}{\mathrm{Pr}_{\varepsilon}} \varepsilon ; k\right) ;,
$$

where

$$
\begin{aligned}
\eta & =\left(2 S^{i j} S_{i j}\right)^{1 / 2} \frac{K}{\varepsilon} \text { and } \\
c_{\varepsilon 1}^{*} & =c_{\varepsilon 1}-\frac{\eta\left(1-\eta / \eta_{0}\right)}{1+\beta \eta^{3}} .
\end{aligned}
$$

The constant $\eta_{0}=4.38$ is the value of $\eta$ in homogeneous shear flow. Accordingly, $c_{\varepsilon 1}=1.42$ is the value of $c_{\varepsilon 1}^{*}$ for homogeneous shear flow. The effect of $c_{\varepsilon 1}^{*}$ is to increase $\varepsilon$ in regions of strong shear. The parameter $\beta$ in the equation for $c_{\varepsilon 1}^{*}$ is determined through the "Law of the Wall" boundary condition. Refer to the discussion in Section $\mathrm{H}$ below.

We have found that neither the standard $K-\varepsilon$ model nor the RNG $K-\varepsilon$ model predicts the mean flow observed in the Purdue experiment. (See the discussion of our results in Section III below.) Refer to Figure 1 and to Figure 2. We believe the reason the $K-\varepsilon$ models do not work well is because they are indifferent to the rotational part of the mean flow. These models are indifferent to the rotational part of the mean flow because the symmetric rate of strain tensor $S_{i j}$ is indifferent to the rotational part of the mean flow. (The rate of stain tensor $S_{i j}$ is an objective tensor.) In addition, the transport equations for $K$ and $\varepsilon$, Eq. (II.7) and Eq. (II.8) or Eq. (II.9), are indifferent to the rotational part of the mean flow. However, the addition of a rigid body rotation to a turbulent flow changes the structure of the turbulent eddies and, so, changes the rate of turbulent transport of momentum. Thus, any physically correct turbulence model should be sensitive to the rotational part of the mean flow. We consider going to Reynolds stress evolution equations because they contain exact terms which are sensitive to the rotational part of the mean flow.

\section{E Reynolds Stress Evolution Equations}

The exact transport equation for $R_{i j}$ has the following form:

$$
\frac{\partial R^{i j}}{\partial t}+C^{i j}=\mathcal{P}^{i j}+\Pi^{i j}-2 \varepsilon^{i j}+J^{i j k} ;,
$$

where

$$
C^{i j}=v^{k} R^{i j}{ }_{i k}
$$

is the convection tensor,

$$
\mathcal{P}^{i j}=-R^{i k} v^{j} ; k-R^{j k} v^{i} ; k
$$

is the production term,

$$
\Pi_{i j}=\frac{1}{\rho} \overline{p^{\prime}\left(v_{i j}^{\prime}+v_{j}^{\prime}{ }_{i}\right)}
$$


Swirl Velocity with RNG K- $\varepsilon$ Model
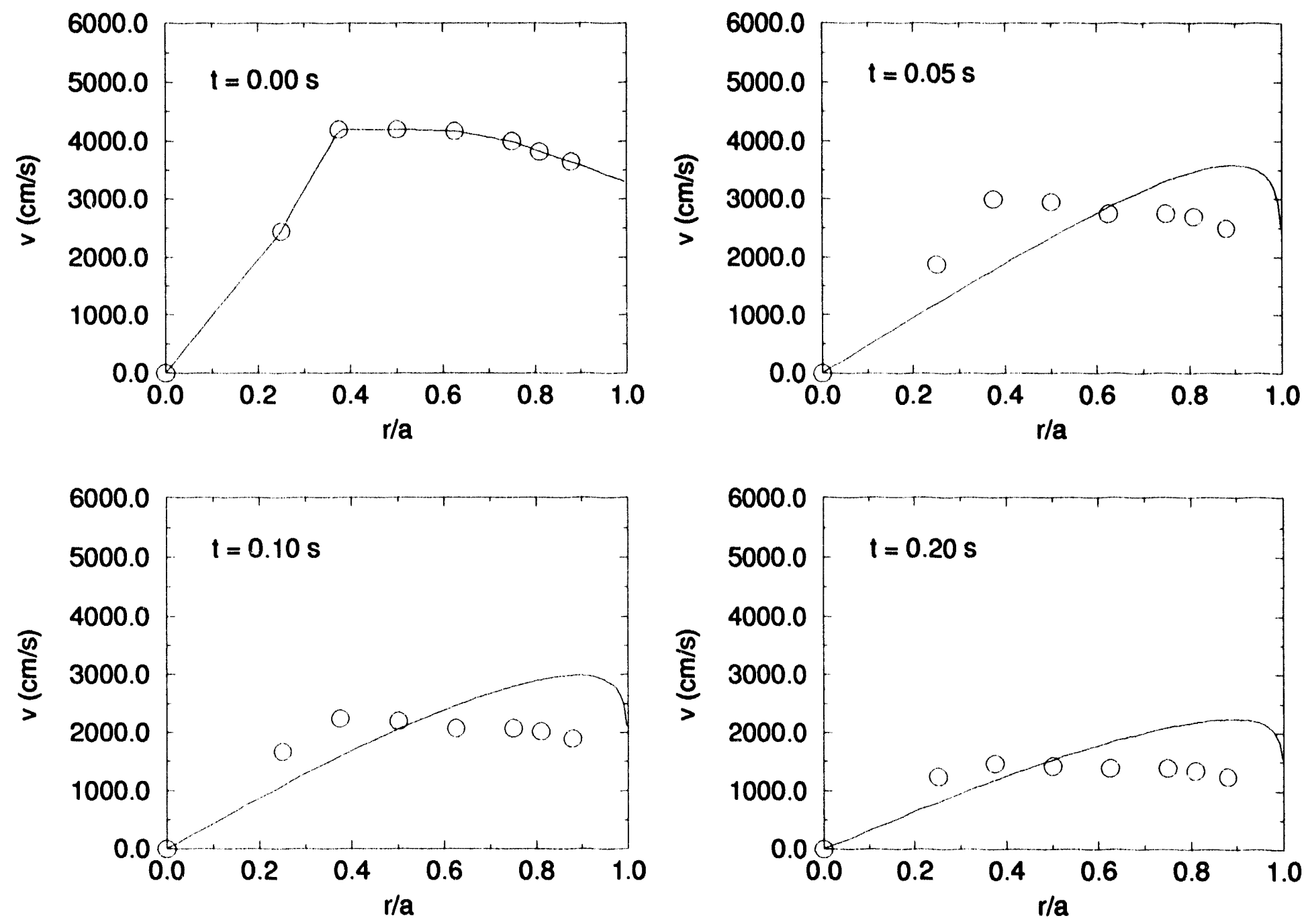

Figure 2: A time sequence of the azimuthal velocity profiles along the central plane as a function of radial position $r$ divided by the cylinder radius $a$. The solid lines are from a one-dimensional calculation and the circles correspond to experimental measurements. The calculation was done using the RNG $K-\varepsilon$ turbulence model. 
is the pressure-strain correlation tensor, and

$$
\varepsilon_{i, j}=\nu \overline{v_{i}^{\prime} ; k v_{j}^{\prime} ; k}
$$

is the dissipation tensor. Finally, the flux of $R_{i j}$ in the kth direction is

$$
J_{i j k}=\frac{1}{\rho}\left(\overline{p^{\prime} v_{j}^{\prime}} \delta_{i k}+\overline{p^{\prime} v_{i}^{\prime}} \delta_{j k}\right)-\overline{v_{i}^{\prime} v_{j}^{\prime} v_{k}^{\prime}}+\nu R_{i j ; k} .
$$

Following Launder et al. ${ }^{6}$, we model the turbulent transport of $R_{i j}$ by a nonlinear diffusion term, namely,

$$
J_{; k}^{i j k}=\left(\frac{3}{2} \frac{c_{\mu}}{\operatorname{Pr}_{K}} \frac{K}{\varepsilon} R^{k l} R_{; l}^{i j}\right) ; k_{i k}
$$

Also following convention, we model the dissipation term with the $\varepsilon$-equation via the relation

$$
\varepsilon_{i j}=\frac{1}{3} \varepsilon \delta_{i j}
$$

For our calculations, we find that the model for $\boldsymbol{\Pi}$ is crucial for accurately computing the mean flow. So, we consider three models for $\boldsymbol{\Pi}$ : the original Launder, Reece, and Rodi model (LRR), ${ }^{6}$ the extension of the LRR model called the "basic model" (LRRb) ${ }^{2}$ (which we made Cialilcan invariant), and the model developed by Speziale, Sarkar, and Gatski (SSG). ${ }^{\top}$

Launder et al. split the model for the pressure-strain correlation into a "slow" part, $\boldsymbol{\Pi}^{(s)}$, and a "rapid" part, $\Pi^{(r)}{ }^{6}$ The slow part is the effect of the fluctuating velocity gradients on the fluctuating pressure and is modeled using Rotta's return to isotropy model ${ }^{8}$

$$
\Pi_{i j}^{(s)}=-c_{1} \frac{\varepsilon}{h}\left(R_{i j}-\frac{2}{3} K \delta_{i j}\right) .
$$

The rapid part describes the coupling of the turbulence with the mean flow through the incompressibility constraint. The LRR model for $\boldsymbol{\Pi}^{(r)}$ is given by

$$
\mathrm{II}_{i j}^{(r)}=-c_{2}\left(\mathcal{P}_{i j}-\frac{1}{3} \mathcal{P}_{k}^{k} \delta_{i j}\right) .
$$

The Galilean invariant version of the LRRb model for $\Pi^{(r)}$ is the same as the LRR model except that $P_{i j}$ is replaced by $P_{i j}-\frac{D R_{i 1}}{D t}$, namely,

$$
\Pi_{i j}^{(r)}=-c_{2}\left[\mathcal{P}_{i j}-\frac{D R_{i j}}{D t}-\frac{1}{3}\left(\mathcal{P}_{k}^{k}-2 \frac{D K}{D t}\right) \delta_{i j}\right]
$$

where $\frac{D R_{i 1}}{D t}$ is the material derivative of $R_{i j}$ given by

$$
\frac{D R_{i j}}{D t}=\frac{\partial R_{i j}}{\partial t}+C_{i j}
$$

This modification turns out to be most important, and we discuss the motivation for it in Section $\mathrm{F}$ below.

In near-wall flows, Launder et al. ${ }^{2}$ make an addition to $\boldsymbol{I}$ to account for wall effects:

$$
\begin{aligned}
\Pi_{i j}^{(w)} & =\left[c_{1}^{\prime} \frac{\varepsilon}{K}\left(R_{k m} n^{k} n^{m} \delta_{i j}-\frac{3}{2} R_{i k} n^{k} n_{j}-\frac{3}{2} R_{k i} n^{k} n_{i}\right)\right. \\
& \left.+c_{2}^{\prime}\left(\Pi_{k m}^{(r)} n^{k} n^{m} \delta_{i j}-\frac{3}{2} \Pi_{i k}^{(r)} n^{k} n_{j}-\frac{3}{2} \Pi_{k j}^{(r)} n^{k} n_{i}\right)\right] f^{2}
\end{aligned}
$$


where $n$ is the unit normal to the wall and $f$ is the ratio of the turbulent length scale to the normal distance to the wall $x_{n}$. This length scale ratio $f$ is given by

$$
f=\frac{\left(c_{l} h\right)^{3 / 2}}{\kappa \varepsilon x_{n}},
$$

where $c_{l}$ is the ratio of $v_{*}^{2}$ to $K$ in the logarithmic region. For the definition of $v_{*}$, see the discussion of the logarithmic region in Section $\mathrm{H}$ below.

Recently, Speziale et al. developed a more sophisticated model for $\boldsymbol{\Pi}^{7}$ than the LRR model. They used various homogeneous turbulent flows, including flows with a rigid-body rotation, to test and calibrate their model. The result of their study is

$$
\begin{aligned}
\mathrm{I}_{i, j}= & -\left(c_{1} \varepsilon+\frac{1}{2} c_{1}^{*} \mathcal{P}_{k}^{k}\right) b_{i, j}+c_{2} \varepsilon\left(b_{i k} b_{j}^{k}-\frac{1}{3} b_{m n} b^{m n} \delta_{i j}\right)+\left(c_{3}-c_{3}^{*} I I^{1 / 2}\right) K S_{i j} \\
& +c_{4} K\left(b_{i}^{k} S_{j k}+b_{j}^{k} S_{i k}-\frac{2}{3} b^{m n} S_{m n} \delta_{i, j}\right)+c_{5} K\left(b_{i}^{k} W_{j k}+b_{j}^{k} W_{i k}\right),
\end{aligned}
$$

where $b_{i j}$ is the anisotropy tensor given by

$$
b_{i j}=\frac{R_{i j}}{2 K}-\frac{1}{3} \delta_{i j},
$$

$I I$ is an invariant of the anisotropy tensor given by

$$
I I=b^{m n} b_{m n},
$$

and $W_{i j}$ is the antisymmetric tensor

$$
W_{i j}=\frac{1}{2}\left(v_{i} i_{j}-v_{j i}\right) .
$$

In their study of the pressure-strain correlation model, the flows had zero convection. However, the convection tensor $C_{i j}$ is important in the flow we are considering. Therefore, next, we discuss the incorporation of $C_{i j}$ into the LRR model for $\boldsymbol{\Pi}$.

\section{F Covariant Modeling of the Pressure-Strain Correlation Tensor}

Launder et al. ${ }^{6}$ proposed that the action of $\boldsymbol{\Pi}^{(r)}$ is 10 make the production of turbulence more isotropic. Accordingly, the reduced form of the original LRR model for $\boldsymbol{\Pi}^{(r)}$ is proportional to the traceless part of $\mathcal{P}$, Eq. (II.19). However, this modeling assumption is not valid for a general coordinate system because it is not based on the covariant forms of $\mathcal{P}$ and $\boldsymbol{C}$.

In terms of the covariant derivative, the covariant forms of $\mathcal{P}^{i j}$ and (iij are

$$
\begin{gathered}
\mathcal{P}^{i j}=-R^{i k} v^{j}, k-R^{j k} v^{i}, k-\left\{\begin{array}{c}
j \\
k l
\end{array}\right\} R^{i k} v^{l}-\left\{\begin{array}{c}
i \\
k l
\end{array}\right\} R^{j k} v^{l} \text { and } \\
C^{i j}=v^{k} R^{i j}, k+\left\{\begin{array}{c}
j \\
k l
\end{array}\right\} R^{i l} v^{k}+\left\{\begin{array}{c}
i \\
k l
\end{array}\right\} R^{j l} v^{k},
\end{gathered}
$$

where we have used the symmetry of $R^{i j}$ in arranging the indices. (Refer to the expression for the production tensor, Eq. (11.12), the expression for the convection tensor, Eq. (11.11), and to the formula for the covariant derivative, Eq. (II.1).) In a general coordinate frame, the last two 
terms in $\mathcal{P}^{i j}$ and $-C^{i j}$ are identical. So, if $\boldsymbol{\Pi}^{(r)}$ acts to make the terms in $\mathcal{P}_{i j}$ more isot ropic, it should act in the same way on the jdentical terms in - $C^{i}$. These ideas were first proposed by Fu et al. ${ }^{9}$ Accordingly, the current basic LRR model for $\boldsymbol{\Pi}^{(r)}$ is proportional to the traceless part of $\left(\mathcal{P}^{i j}-C^{i j}\right)$. To make the model for $\Pi^{(r)}$ Galilean invariant, ( ${ }^{i, j}$ should be replaced by the material time derivative of $R^{i j}$. Refer to the LRRb model for $\Pi^{(r)}$ given in Ey. (II.20). (Apparently, Launder failed to see this point. ${ }^{2}$ )

In contrast to the constitutive relations found in continuum mechanics, this model is not "objective" in the sense that when the tensor $\left(\mathcal{F}^{i j}-D R^{i j} / D t\right)$ is transformed to a rotating framc, there is an extra "Coriolis" term. It is the combination $\left(\mathcal{P}^{i j}+D R^{i j} / D t\right)$ which is objective. Actually, that there should be a difference between turbulence models and constitutive relations found in continuum mechanics makes sense. The turbulent transport of momentum is sensitive to the presence of rotation in the mean flow; and, so, any turbulence model should be sensitive to the rotational part of the mean flow. On the other hand, the elastic stress tensor, for example, is related to the deformation of a material element and is unaffected by rotational motion.

In summary, the combination $\left(\mathcal{P}^{i j}-D R^{i j} / D t\right)$ should be used in modeling the rapid part of the pressure-strain correlation tensor because $\mathcal{P}^{i j}$ and $-C^{i j}$ have terms that are identical, as revealed when the equations are written in covariant form. The combination $\left(\mathcal{P}^{i, I}-D R^{i j} / D t\right)$ is a tensor because we use covariant derivatives, not because of the grouping of terms.

\section{G Cylindrical Coordinates}

We now discuss the equations of motion in cylindrical coordinates since these are the equations we used in our calculations. We take the cylindrical coordinates to be $r, \phi, z$, where $z$ is along the cylinder axis of symmetry, $r$ is the radial distance to the wall, and $\phi$ is the azimuthal angle. For simplicity, we neglect the finite height of the cylinder and say that there is no $z$ dependence. Because of azimuthal symmetry, we assume that the variables do not depend on $\phi$. As a result, the variables only depend on $r$ and time $t$. We define the cylindrical components of $v$ as $u, v, w$. From the incompressibility constraint, $u=0$ to avoid a singularity at the origin. Without further loss of generality, we set $w=0$. Then the momentum equations, Eq. (II.5), reduce to

$$
\frac{\partial v}{\partial t}=-\frac{\partial C}{\partial r}-2 \frac{C}{r}
$$

where $C=R_{r \phi}$.

The $K$ and $\varepsilon$ equations reduce to

$$
\begin{aligned}
\frac{D K}{D t} & =\mathcal{P}_{K}-\varepsilon+\mathcal{D}_{K}, \\
\frac{D E}{D t} & =+c_{\varepsilon 1} \frac{\varepsilon}{K} \mathcal{P}_{K}-c_{\varepsilon 2} \frac{\varepsilon^{2}}{K}+\mathcal{D}_{\varepsilon},
\end{aligned}
$$

where the various terms are expressed in cylindrical coordinates in Figure 3. Note that the diffusivity in $\mathcal{D}_{\varepsilon}$ is $\nu_{t}$ instead of $\nu_{r r}$. We use $\nu_{t}$ in $\mathcal{D}_{\varepsilon}$ for ease in satisfying the "Law of the Wall" boundary conditions. Refer to Section $\mathrm{H}$ below.

Similarly, the Reynolds Stress equations, Eq. (11.10), can be written as

$$
\frac{D A}{D t}=\mathcal{P}_{A}+\Pi_{A}+\mathcal{D}_{A}-\frac{4}{3} \varepsilon
$$




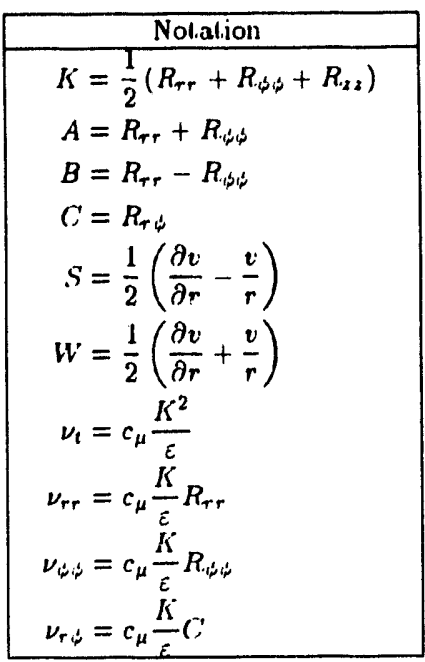

Malerial Derivalive

$$
\begin{aligned}
& \frac{D E}{D}=\frac{\partial \varepsilon}{\partial l} \\
& \frac{D K}{D}=\frac{\partial K}{\partial !} \\
& \frac{D A}{D}=\frac{\partial A}{\partial !} \\
& \frac{D B}{D}=\frac{\partial B}{\partial 1}+4 C(S-W) \\
& \frac{D C}{D}=\frac{\partial C}{\partial l}-B(S-W)
\end{aligned}
$$

$$
\begin{aligned}
& \text { Produclion Term } \\
& \mathcal{P}_{K}=-2 C S \\
& P_{A}=-4 C \cdot S \\
& P_{B}=4 C W \\
& P_{C}=-A S-B W
\end{aligned}
$$

$$
\begin{aligned}
& D_{t}=\frac{1}{r} \frac{\partial}{\partial r}\left(r \frac{\nu_{t}}{P_{r_{t}}} \frac{\partial \tau}{\partial r}\right) \\
& D_{k}=\frac{1}{r} \frac{\partial}{\partial r}\left(r \frac{\nu_{r r}}{\operatorname{Pr}_{k}} \frac{\partial K}{\partial r}\right) \\
& D_{A}=\frac{1}{r} \frac{\partial}{\partial r}\left(r \frac{\nu_{r r}}{P_{r_{k}}} \frac{\partial A}{\partial r}\right) \\
& D_{B}=\frac{1}{r} \frac{\partial}{\partial r}\left(r \frac{\nu_{r r}}{\operatorname{Pr}_{K}} \frac{\partial B}{\partial r}-4 \frac{\nu_{r_{r}}}{\operatorname{Pr}_{K}} C\right)-4 \frac{\nu_{r}}{\operatorname{Pr}_{K}} \frac{1}{r} \frac{\partial C}{\partial r}-4 \frac{\nu_{r j}}{\operatorname{Pr}_{K}} \frac{B}{r^{2}}
\end{aligned}
$$

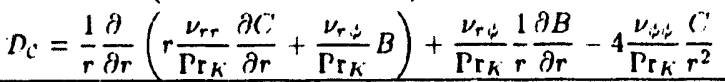

Figure 3: A summary of the terms in the Reynolds stress equations in cylindrical coordinates under the assumptions discussed in the text. 


$$
\begin{aligned}
& \frac{D B}{D x}=\mathcal{P}_{B}+\Pi_{B}+\mathcal{D}_{B}, \text { and } \\
& \frac{D C}{D t}=\mathcal{P}_{C}+\mathrm{II}_{C}+\mathcal{D}_{C},
\end{aligned}
$$

where, again, the various terms are expressed in cylindrical coordinates in Figure 3 . Figure 4 shows a summary of the various pressure-strain correlation models in cylindrical coordinates.

The variable $A$ is the turbulent kinetic energy in the $x y$-plane, the plane perpendicular to the axis of symmetry. The amount of anisotropy in the components of the Reynolds stress tensor in the $x y$-plane is related to $B$. The variable $S$ is the shear of the mean flow, and the variable $W$ is the vorticity. From the production term box in Figure 3, we see that vorticity produces anisotropy while shear creates turbulent kinetic energy. Also, from the material derivative box in Figure 3 , we see that convection of $B$ and $C$ by the azimuthal velocity occurs even though $B$ and $C$ are independent of $\phi$. Note the appearance of identical terms in the convection and production of $B$ and $C$.

\section{H Turbulent Boundary Layer Treatment}

We close out this section with a discussion of the boundary conditions that arise due to the turbulent boundary layer at the cylinder wall. For computational efficiency and because our turbulence models are only valid at high Reynolds number, we do not resolve the viscous sublayer. Instead, we use the "Law of the Wall" to determine appropriate boundary conditions for the turbulence equations.

The derivation of the Law of the Wall is based on the idealization of a steady turbulent. flow directed parallel to an infinite plane. In this section, we take the $x_{1}=x$ coordinate direction to be along the flow and the $x_{2}=y$ coordinate direction to be normal to the wall. As a result of the symmetry in the $x z$-plane, the flow can only vary in the $y$-direction. With these assumptions, onc can derive the logarithmic velocity profile by using only dimensional reasoning. ${ }^{10}$

Based on dimensional analysis, the velocity gradient takes the asymptotir form

$$
\frac{d u}{d y}=\frac{v_{*}}{\kappa y},
$$

where $\kappa$ is the von Karman constant, experimentally determined to be

$$
\kappa=0.4
$$

and $v_{*}$ is related to the wall shear stress $\sigma$ through the definition

$$
\sigma=\rho v_{*} v_{*} .
$$

By integrating Eq. (II.25) with respect to $y$, one finds that the velocity has a logarithmic profile

$$
u=v_{*}\left[\frac{1}{\kappa} \log \left(\frac{v_{*} y}{\nu_{0}}\right)+B\right]
$$

valid for $v_{*} y / \nu_{0} \gg 1$. The parameter $\nu_{0}$ is the molecular viscosity and $B$ is an experimental constant equal to 5.5 for smooth walls.

The mean energy flux density to the wall is given by $q=\sigma u$. $A$ s the flux approaches the wall, $q$ decreases because turbulent energy is being dissipated. The rate of dissipation per volume is given 


\begin{tabular}{|c|}
\hline LRR Model for $\Pi$ \\
\hline$c_{1}=1.8, c_{2}=0.6$ \\
\hline$\Pi_{A}=-c_{1} \frac{\hat{c}}{K}\left(A-\frac{4}{3} K\right)+\frac{4}{3} c_{2} C S S$ \\
$\Pi_{R}=-c_{1} \frac{\bar{c}}{K} B-4 c_{2} C^{\prime} W$ \\
$\Pi_{C}=-c_{1} \frac{\tilde{\varepsilon}}{K} C^{\prime}+c_{2}(A S+B W)$ \\
\hline
\end{tabular}

\begin{tabular}{|c|}
\hline LRR.b Model for $\boldsymbol{\Pi}$ \\
\hline$c_{1}=1 . \overline{8}, c_{2}=0.6$ \\
$\Pi_{A}=-c_{1} \frac{\bar{C}}{K}\left(A-\frac{4}{3} K\right)+\frac{4}{3} c_{2} C S+c_{2}\left(\frac{\partial A}{\partial l}-\frac{4}{3} \frac{\partial K}{\partial l}\right)$ \\
$\Pi_{B}=-c_{1} \frac{\bar{c}}{K} B+4 c_{2} C^{\prime}(S-2 W)+c_{2} \frac{\partial B}{\partial l}$ \\
$\Pi_{C}=-c_{1} \frac{\bar{c}}{K} C^{\prime}+c_{2}[A S-B(S-2 W)]+c_{2} \frac{\partial C^{\prime}}{\partial l}$ \\
\hline
\end{tabular}

\begin{tabular}{|c|c|}
\hline Wall Correclion Funclions & \\
\hline$c_{1}=1.8, c_{2}=0.6, c_{1}^{\prime}=0 . \overline{1}, c_{2}^{\prime}=0.3$ & \\
\hline$f=\frac{\left(c_{1} k\right)^{3 / 5}}{\kappa \varepsilon y}, c_{l}=0.26$ & \\
\hline$\Pi_{A}^{(u)}=-c_{1}^{\prime} \frac{\bar{c}}{K} R_{r r}+2 c_{2} c_{2}^{\prime}\left(\frac{1}{3} C \cdot S-C W\right)$ & $f^{2}$ \\
\hline$\Pi_{R}^{(w)}=-3\left[c_{1}^{\prime} \frac{\varepsilon}{K} R_{r r}+2 c_{2} c_{2}^{\prime}\left(\frac{1}{3} C S-C w\right.\right.$ & \\
\hline$\Pi_{C}^{(w)}=-\frac{3}{2}\left[c_{1}^{\prime} \frac{\ddot{c}}{K} C+c_{2} c_{2}^{\prime}(A S+B W)\right] f^{2}$ & \\
\hline
\end{tabular}

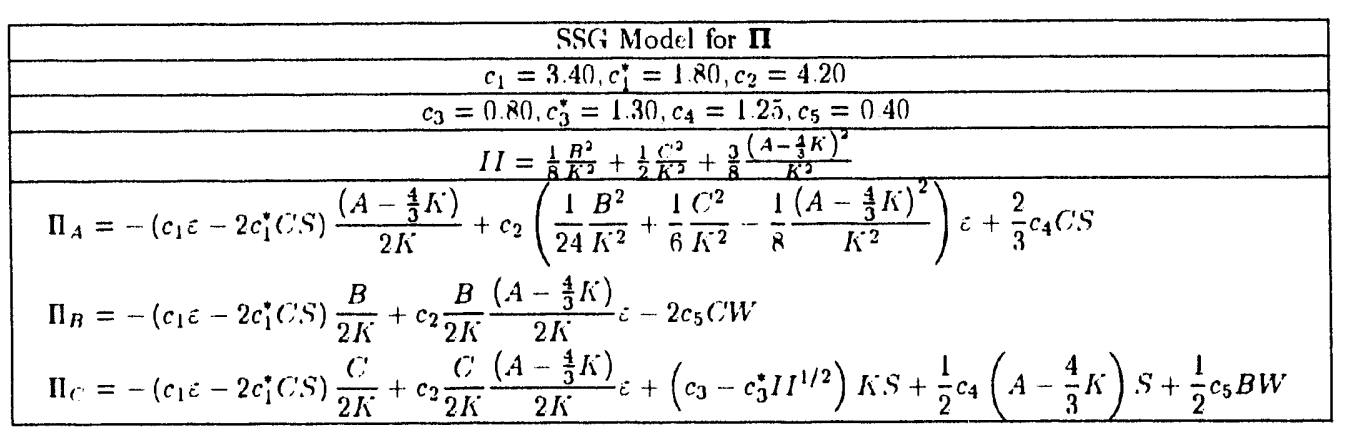

Figure 4: A summary of the various pressure-strain correlation models in cylindrical coordinates under the assumptions discussed in the text. 
by $d q / d y$. Dividing this result by $\rho$ yields the dissipation rate per mass $\varepsilon$, namely,

$$
\varepsilon=\frac{v_{*}^{3}}{\kappa y} .
$$

These results for $u$ and $\varepsilon$ hold for any turbulent boundary layer whose thickness is small compared to the finite dimensions of the container. Moreover, the Law of the Wall is well established experimentally and should be contained in any turbulence model.

In the logarithmic region, where diffusion and time variation can be neglected, the Reynolds stress evolution equations, Eq. (II.10), reduce to

$$
P_{i j}+\Pi_{i j}-\frac{2}{3} \delta_{i j} \varepsilon=0
$$

From the above analysis, the only nonzero component of the velocity gradient $v_{i, j}$ is

$$
v_{1,2}=S=\frac{v_{*}}{\kappa y} .
$$

For this velocity gradient tensor, the production tensor is of the form

$$
\frac{P_{i j}}{\varepsilon}=f_{i j}\left(b_{i j}, \frac{S h}{\varepsilon}\right)
$$

from Eq. (II.12). Likewise, all the models for $\Pi_{i j}$ that we consider have this same asymptotic form

$$
\frac{\Pi_{i j}}{\varepsilon}=g_{i j}\left(b_{i j}, \frac{S h}{\varepsilon}\right) \text {. }
$$

Refer to Eq. (II.18), Eq. (II.19), Eq. (II.21) with $f=1$, and Eq. (II.22). In the logarithmic region, LRRb is identical with LRR since the time variation and convection of $R_{i j}$ are negligible.

Taking the trace of Eq. (11.28) gives

$$
\varepsilon=\mathcal{P}_{K}
$$

where $\mathcal{P}_{K}=(1 / 2) P_{i}^{i}$ is the production rate of turbulent energy. This result is due to the flow being nearly stationary in a turbulent boundary layer. In our coordinate system,

$$
\mathcal{P}_{K}=-R_{12} v_{1,2}=-2 h b_{12} S,
$$

and

$$
\frac{S K}{\varepsilon}=\frac{1}{\left(-2 b_{12}\right)}
$$

As a result, $P_{i j} / \varepsilon$ and $\Pi_{i j} / \varepsilon$ are both equal to some function of $b_{i j}$. So, the Reynolds stress evolution equations reduce to a set of algebraic equations

$$
\frac{P_{i j}}{\varepsilon}+\frac{I_{i j}}{\varepsilon}-\frac{2}{3} \delta_{i j}=0
$$

that we can solve for $b_{i j}$ independent of $\varepsilon \cdot{ }^{11}$ In summary, the system of Reynolds stress equations, Eq. (II.30), yields numerical values for $b_{i j}$ depending on the closure for the pressure-strain correlation tensor.

The numerical values for $b_{i j}$ in the logarithmic region have been determined experimentally. Unfortunately, the experimental values for $b_{i j}$ have a considerable variation. For example, the 
values for $-b_{12}$ range from 0.13 to 0.17 . This variation in the experimental values for $b_{12}$ is reflected in the values for $b_{12}$ obtained from turbulence models by solving the system of equations Eq. (1I.30) for $b_{i j}$. (Refer to Table 1.)

Since $R_{12}$ is the shear stress, we can relate $R_{12}$ to $v_{*}$ according to

$$
R_{12}=-v_{*} v_{*}=-\frac{\sigma}{\rho} \text {. }
$$

Then, the definition of $b_{12}$ gives a relation between $K$ and $r_{*}$

$$
K=\frac{v_{*} v_{*}}{\left(-2 b_{12}\right)}
$$

At the same time, the standard $\varepsilon$-equation, Eq. (II.8), reduces to an ordinary differential equation

$$
\frac{1}{\varepsilon^{2}} \frac{d}{d y}\left[\frac{1}{\varepsilon} \frac{d}{d y}\right]=\frac{1}{h^{3}} \frac{\operatorname{Pr}_{\varepsilon}}{c_{\mu}}\left(c_{\varepsilon 2}-c_{\varepsilon 1}\right) .
$$

In order for Eq. (II.32) to have Eq. (II.27) as a solution it is necessary that

$$
\kappa^{3}=\operatorname{Pr}_{\varepsilon}\left(c_{\varepsilon 2}-c_{\varepsilon 1}\right) \frac{v_{*}^{6}}{c_{\mu} \kappa^{2}} .
$$

By substituting Eq. (II.31) into Eq. (II.33), we obtain a relation between $\operatorname{Pr}_{\varepsilon}$ and $b_{12}$, namely,

$$
\frac{1}{\operatorname{Pr}_{\varepsilon}}=\left(c_{\varepsilon 2}-c_{\varepsilon 1}\right) \frac{\left(-2 b_{12}\right)^{3}}{c_{\mu} \kappa^{2}} \text {. }
$$

So, the $\varepsilon$ Prandlt number is determined by the value of $b_{12}$ given values for $c_{\varepsilon 1}, c_{\varepsilon 2}, c_{\mu}$, and $\kappa$ the Von Karman constant. Refer to Table 1 to see how $\operatorname{Pr}_{s}$ varies with $b_{12}$.

In contrast, in the RNG theory, $\mathrm{Pr}_{\varepsilon}$ is determined by the analysis and the parameter $\beta$ is determined by the Law of the Wall. ${ }^{3}$ Recall that in the RNG $\varepsilon$-equation, Fq. (II.9), $c_{\varepsilon 1}$ is no longer a constant but is a function of the dimensionless shear $\eta$

$$
\begin{aligned}
\eta & =\frac{\left(2 S_{i j} S_{i j}\right)^{1 / 2} K}{\varepsilon} \text { and } \\
c_{\varepsilon 1}^{*} & =c_{\varepsilon 1}-\frac{\eta\left(1-\eta / \eta_{0}\right)}{1+\beta \eta^{3}},
\end{aligned}
$$

where $c_{\varepsilon 1}=1.42$ is the value of $c_{\varepsilon 1}^{*}$ for homogeneous shear flow, where $\eta=\eta_{0}$. Eq. (II.34) is still valid with $c_{\varepsilon 1}$ replaced by $c_{\varepsilon 1}^{*} 3$. The parameter $\beta$ is determined by solving Fq. (II.34) for $c_{\varepsilon 1}^{*}$ and inverting Eq. (11.35) for beta. Refer to Table 2 to see how $\beta$ varies with $b_{12}$.

From Table 2, we see that the value of $\beta$ for the LRRb model is a small negative number which is unphysical since there can be a singularity in $c_{e 1}^{*}$, Eq. (II.35). Consequently, there is an inconsistency between the constants in the LRRb model and the constants in the RNG $\varepsilon$-equation. This is not surprising since the LRRb model was calibrated for $b_{12}=-0.13$, while the RNG $K-\varepsilon$ gives a value of $b_{12}$ equal to -0.15 . One ad hoc way of circumventing this problem is to treat $\beta$ as an adjustable constant. So for the LRRb model, we input $\beta$ to determine $c_{e 1}^{*}$. Then we vary $\mathrm{Pr}_{\varepsilon}$ according to Eq. (II.34), (with $c_{\varepsilon 1}$ replaced by $c_{\varepsilon 1}^{*}$ ), to satisfy the Law of the Wall boundary condition. 


\section{Comparison with Experiment}

\section{A Introduction}

In this section, we discuss the results of applying different turbulence models to the swirling flow in the Purdue experiment. We find that a combination of the Reyuolds stress equations with the RNG $\varepsilon$-equation works best.

\section{B $\quad K-\varepsilon$ Models}

Currently, KIVA ${ }^{12}$, a computer program for chemically reactive flows, has a standard $k-\varepsilon$ turbulence model. Figure 1 shows a comparison between experimentally measured azimuthal velocity profiles and velocity profiles computed using the $k-\varepsilon \operatorname{model}$. The $h-\varepsilon$ results predict an approximately rigid-body rotation velocity profile until the flow fecls the turbulent boundary layer. In stark contrast, the experimental results indicate that the flow is like a rigid-body rotation only near the core of the cylinder. Since the computed volocity profile is nearly wheel flow, the computed mean flow has little shear in the interior of the cylinder. However, the experimentally measured mean velocity shows strong shearing in the interior of the cylinder. Since production of turbulent kinetic energy is directly related to the mean flow shear ( production term box in Figure 3 ), the computed mean flow predicts a small production of turbulence compared to the experimentally measured mean flow (except near the walls).

In order to show what is lost by doing a one-dimensional calculation, Figure 1 shows both a two-dimensional calculation using KIVA ${ }^{12}$ and a one-dimensional calculation using a test computer code. The one-dimensional calculation does not capture the loss of momentum at the top and bottom turbulent boundary layers; and so, the one-dimensional computed velocity profile is about $10 \%$ high compared to the KIVA results. Still the one-dimensional calculation captures a big part of the flow; the computed velocity profiles have the same shape.

For the same calculation, we show the results for the turbulence intensity in Figure 5. The experimentally reasured turbulence intensity is actually the rms value of the fluctuating part of the azimuthal velocity. So, to make this comparison, we have to assume that the Reynolds stress tensor is isotropic, which is not true. Still, the computed results are in good agreement. with the experimentally measured turbulence intensity. As with the azimuthal velocity profile, the turbulence intensity computed using the one-dimensional calculation is about $5 \%$ high compared to the KIVA results because the one-dimensional calculation does not contain the loss of turbulence energy in the top and bottom boundary layers. Because the computed results give good agreement for the wrong level of turbulence energy production, we believe the agreement for the furbulence intensity is more a reflection of the correctness of the "Law of the Wall" boundary conditions than of the correctness of the $K-\varepsilon$ model.

For completeness, we considered the RNG $K-\varepsilon$ model. Refer to the discussion of the RNG $K-\varepsilon$ equations in Section D. The computed results using the RN(: $h-\varepsilon$ model are very comparable to the results using the standard $K-\varepsilon$ model as shown in Figure 2 for the azimuthal velocity profile. We expect the standard $K-\varepsilon$ model and the RNC $K-\varepsilon$ model to give similar results since they are both indifferent to the rotational part of the mean flow, which is unphysical. 
Turbulence Intensity with K- $\varepsilon$ Model
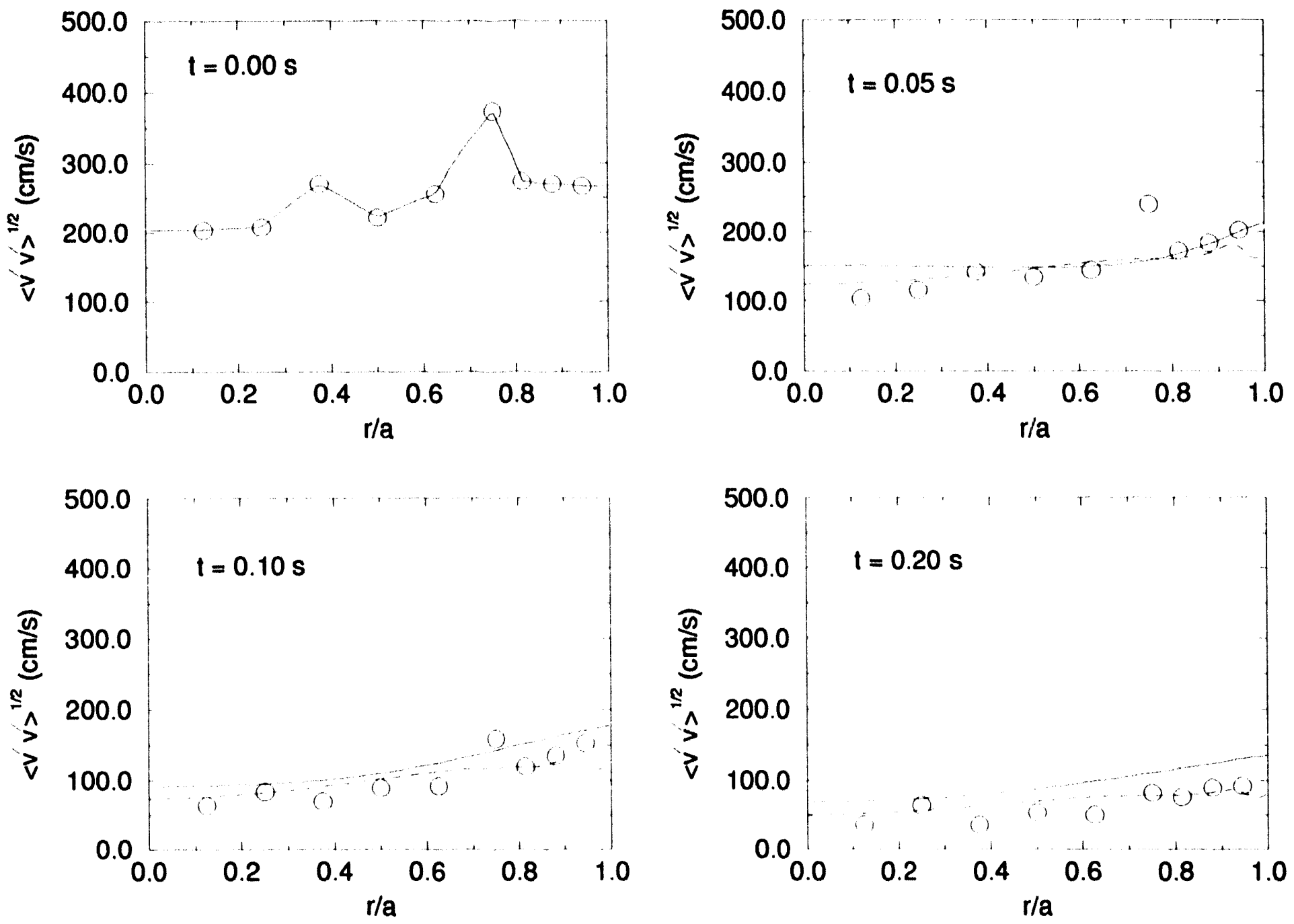

Figure 5: A time sequence of the turbulence intensity curves along the central plane as a function of radial position $r$ divided by the cylinder radius $a$. The turbulence intensity is the ans value of the fluctuating part of the azimuthal velocity. 'The solid lines are from a one-dimensional calculation, the dashed lines are from a two-dimensional KIVA calculation, and the circles correspond to experimental measurements. Both calculations were done using the standard $K-\varepsilon$ model. 


\section{Reynolds Stress Models}

We next consider turbulence models for the Reynolds stress evolution equations. Figure 6 shows the same calculation as above except that the turbulence model is the launder, Recce, and Rodi (LRR) Reynolds stress evolution equation, ${ }^{6} \mathrm{Eq}$. (1I.10), Eq. (II.18), and Eq. (II.19). Though the turbulence modeling is primitive, the calculated results show rigid-body rotation only near the core of the cylinder (like the experimental results). Still, a large discrepancy remains between the calculated and experimental mean flow near the peak of the computed velocity.

For comparison, we also tried the SSG pressure-strain model, Eq. (11.22). As Figure 7 shows, the results using the SSG model are very comparable to the results using the LRR model for the azimuthal velocity profile (Figure 6 ). The reason the SS( $i$ model does not work well is because it does not contain the crucial convection tensor $C_{i j}$. Refer to the discussion in Section $\mathrm{E}$.

To obtain good agreement with experiment, we incorporate a simple modification to the LR R model of the pressure-strain term proposed by Fu $\epsilon$ al. ${ }^{3}$ which we refer to as the LRRb model, Eq. (II.20). See the discussion in Section F. Figure 8 shows the results of a calculation done using the LRRb model. Good agreement is obtained between the calculated and experimental mean velocity profile without adjusting the constants of the model.

However, when we consider the computed turbulence intensity shown in Figure 9, we see that the computed turbulence intensity is too high compared to the experimentally measured turbulence intensity. Because we are now obtaining good agreement with the meall flow, we are producing more turbulence compared to a calculation done with a $h-\varepsilon$ model. So for about the right amount of turbulence energy production, the standard $\varepsilon$-equation predicts a wrong level of turbulence intensity.

To reduce the turbulence intensity, we considered the RNG s-equation, Eq. (II.9), since it has an extra term which increases $\varepsilon$ in regions of high shear. When we use the l.R Rh Reynolds stress equations in conjunction with the RNG s-equation, we are able to obtain good agreement both with the mean flow, Figure 10, and with the turbulence intensity, Figure 11. However, we did treat $\beta$ as an adjustable parameter. We found that the turbulence model with $3=0.040$ gave both the azimuthal velocity profiles and the turbulence intensity curves with reasonable accuracy. for a given input value of $\beta$, the $\varepsilon$ Prandlt number, $\mathrm{Pr}_{\varepsilon}$. was fixed by the Law of the Wall boundary conditions as explained in Section $H$.

\section{Conclusions}

In summary, we have shown how the incorporation of a Reynolds stress evolution equation leads to good agreement between the calculated and the experimentally measured mean velocity profile in the Purdue experiment. No model of the $K-\varepsilon$ type gave good agreement. To obtain this agreement, we used Launder's basic model. ${ }^{2}$ which we modified to be Cialilean invariant. (Refer to the discussion in Section F.) The crucial change in the LRRb over the original LRR model is the addition of the convection tensor to the model for the rapid part of the pressure-strain correlation tensor. However, when we correctly calculate the mean flow, the standard s-equation predicts a high turbulence intensity relative to the experimental measurements.

So, we considered using the $\mathrm{R} N \mathrm{NG}$-equation since it contains a term which increases $\varepsilon$ in regions of strong shear. We found that using the LRRb Reynolds equations in conjunction with the RNG $\varepsilon$-equation yields good agreement between the calculated and the experimentally measured mean 
Swirl Velocity with LRR Model
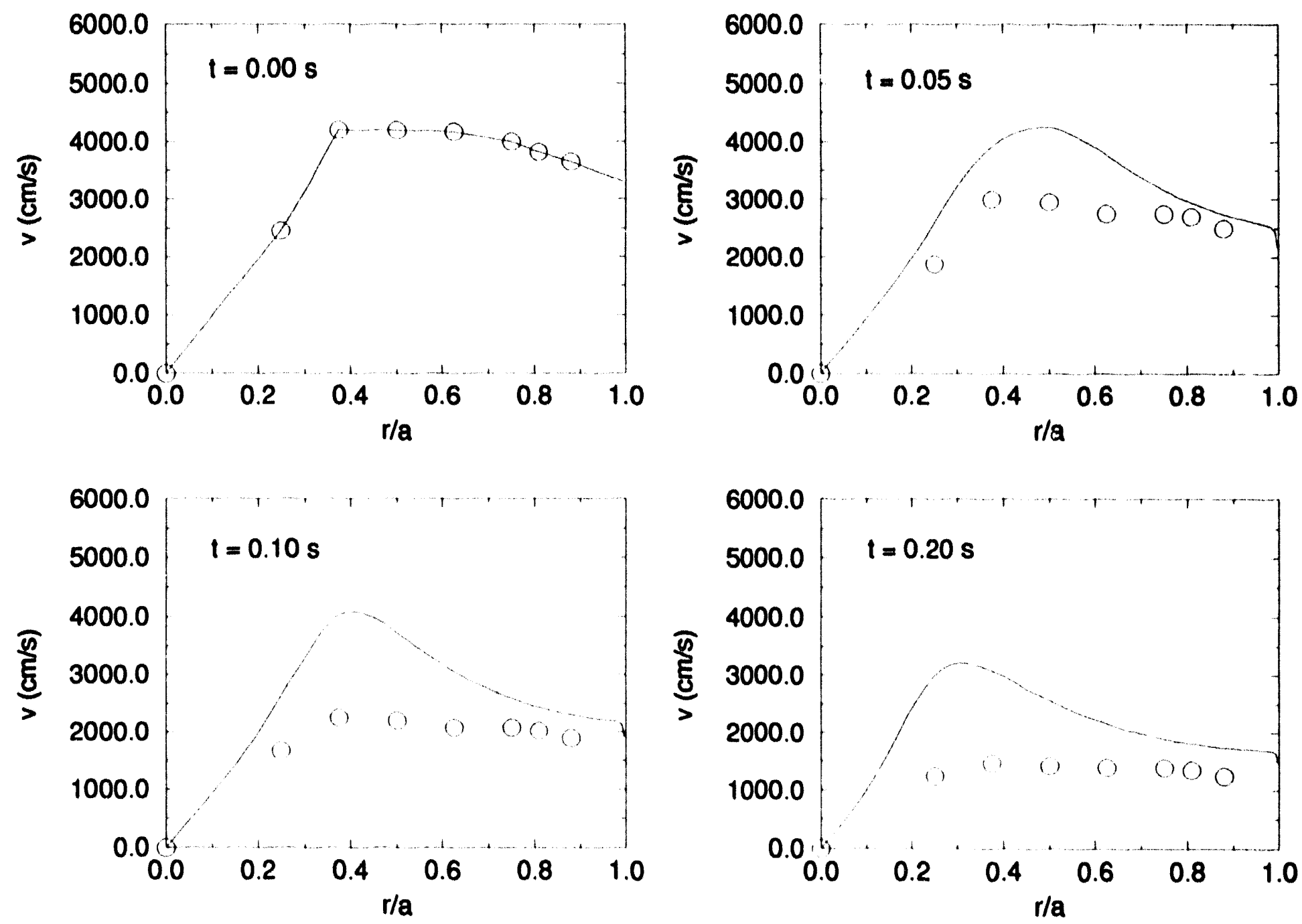

Figure 6: A time sequence of the azimuthal velocity profiles along the central plane as a function of radial position $r$ divided by the cylinder radius $a$. 'The solid lines are from a one-dimensional calculation, and the circles correspond to experimental measurements. 'The calculation was done using the LRR Reynolds stress equations. 
Swirl Velocity with SSG Model
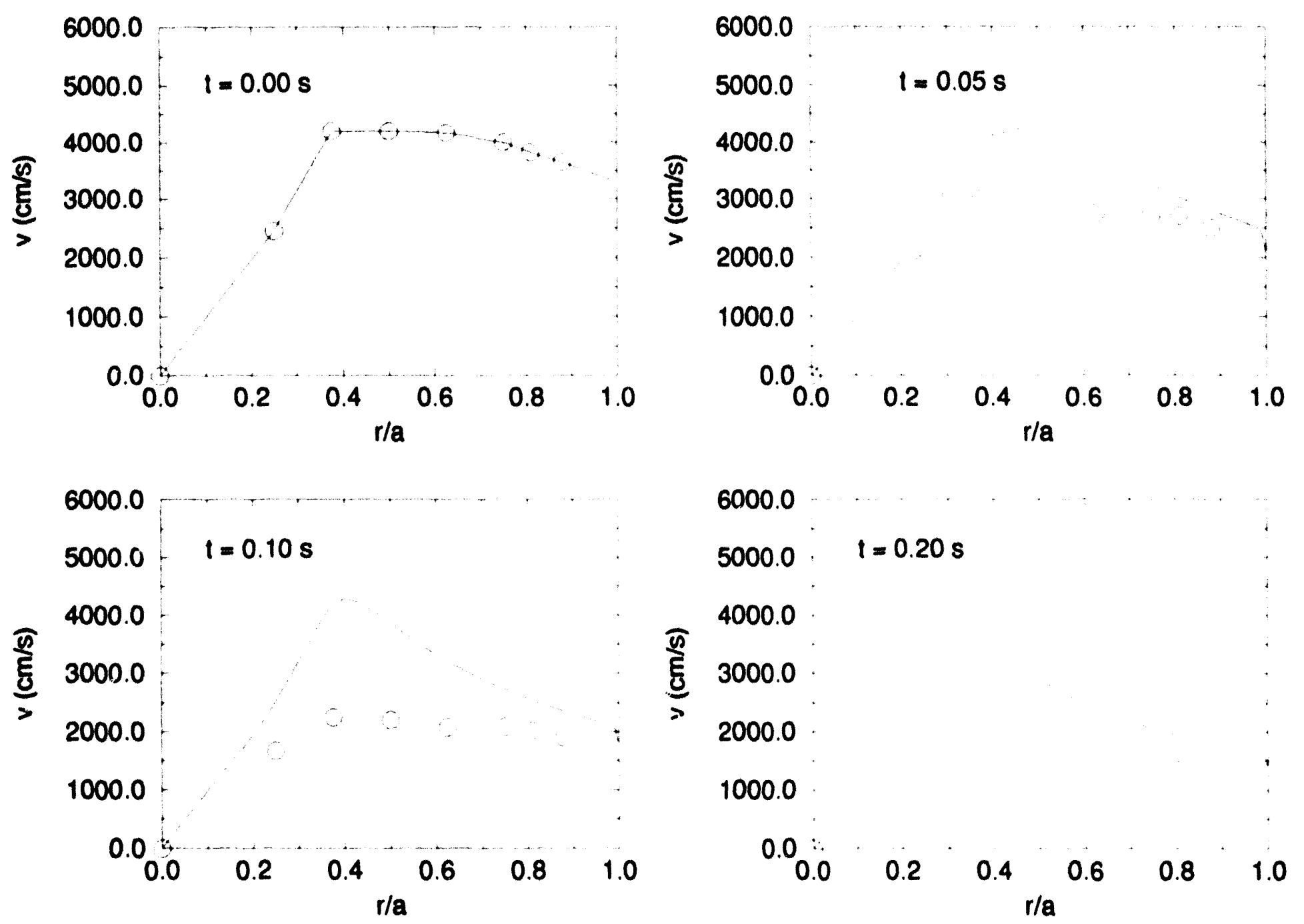

Figure 7: A time sequence of the azimuthal velocity profiles along the cent ral plane as a function of radial position $r$ divided be the cylinder radius a. The solid lines are from a one-dimensional calculation, and the circles rorrespond to experiment al measurements. The calculation was done using the SSC $i$ pressure-strain model. 
Swirl Velocity with LRRb Model
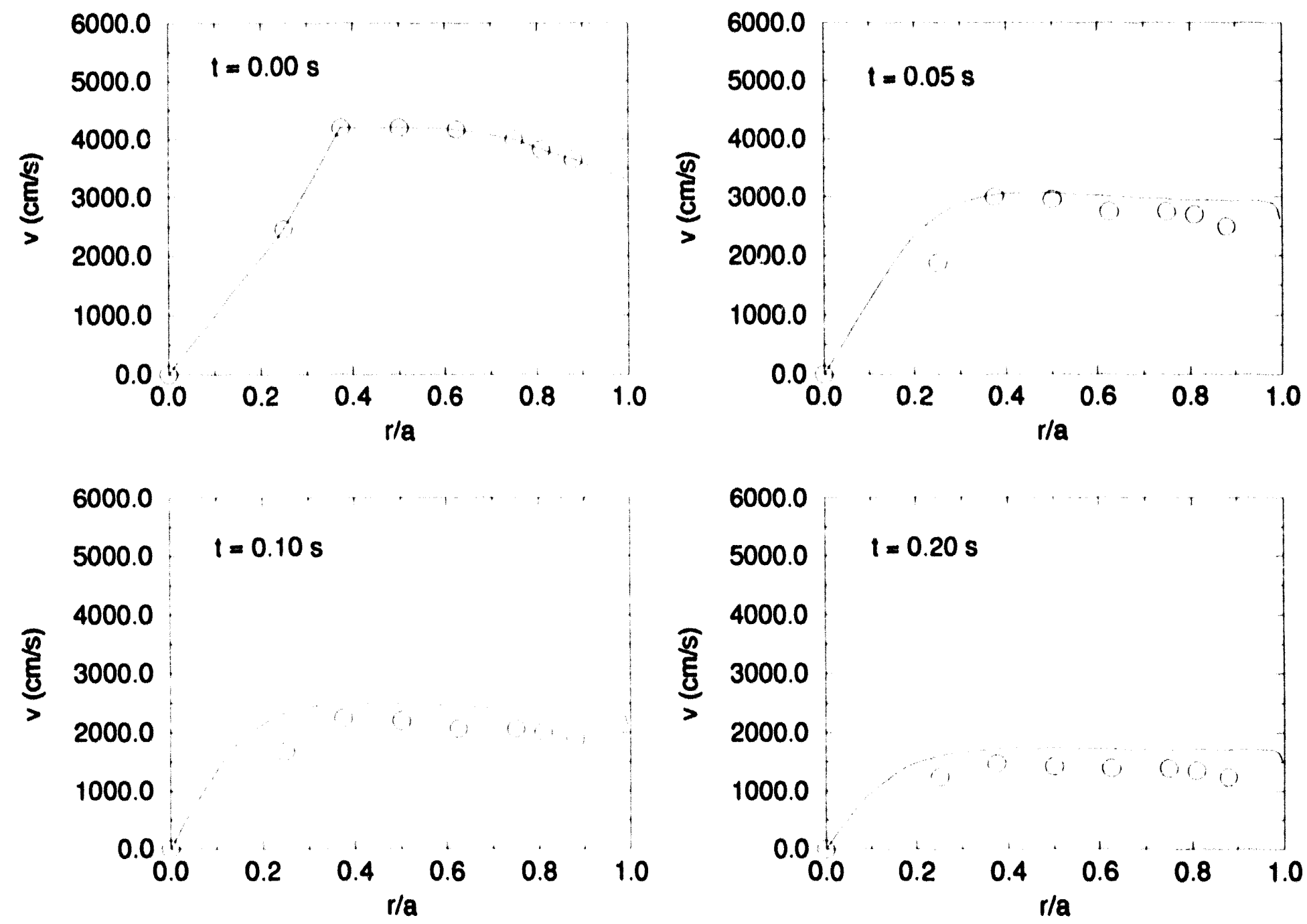

Figure 8: A time sequence of the azimuthal velocity profiles along the cent ral plane as a function of radial position $r$ divided by the cylinder radius $a$. 'The solid lines are from the calculation, and the circles correspond to experimental measurements. The calculation was done using the L,RRL Reynolds stress equations in conjunction with the standard E-equation. 
Turbulence Intensity with LRRb Model
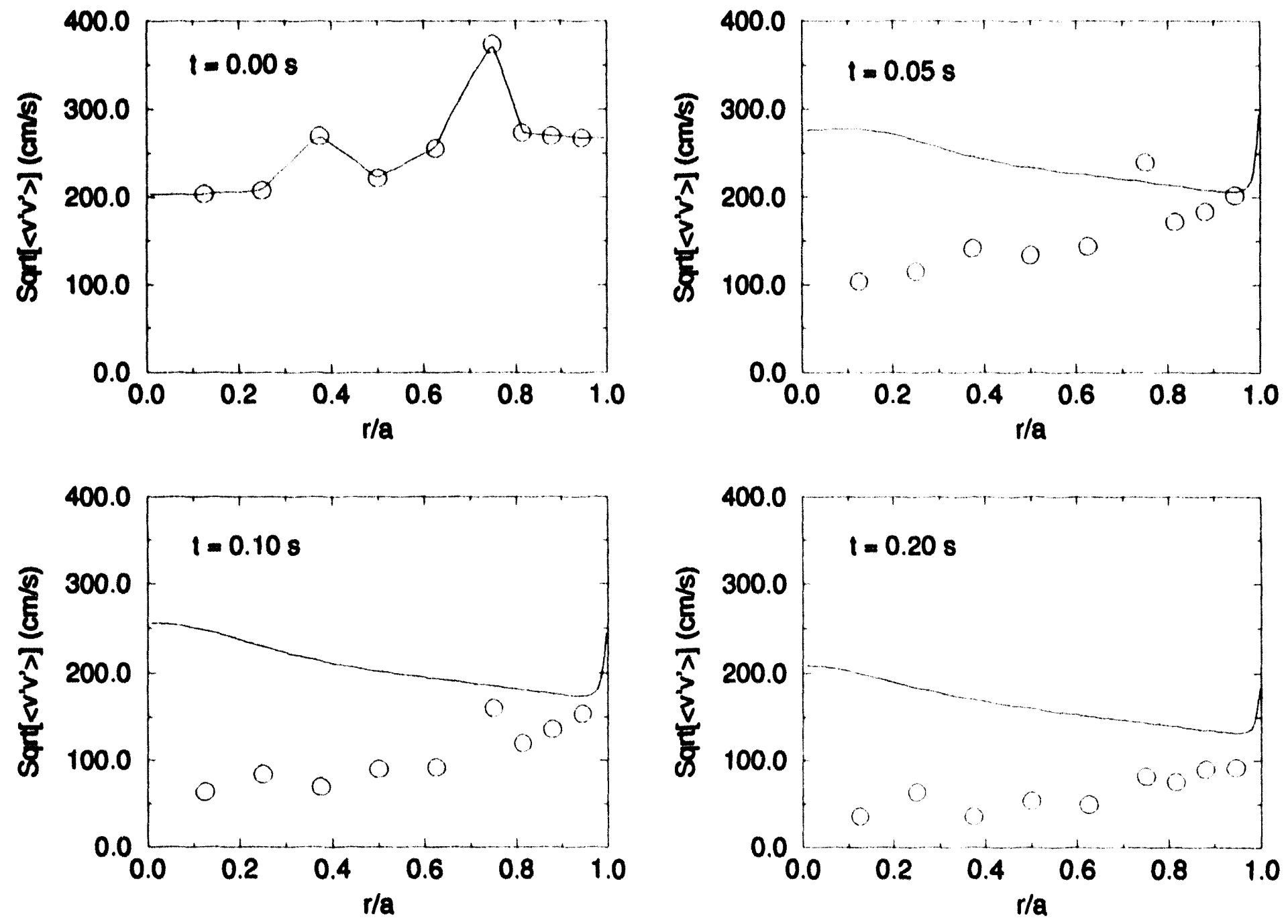

$\mathrm{N}=100$

Figure 9: A time sequence of the turbulence intensity curves along the central plane as a function of radial position $r$ divided by the cylinder radius $a$. The turbulence intensity is the rms value of the fluctuating part of the azimuthal velocity. The solid lines are from a one-dimensional calculation, and the circles correspond to experimental measurements. The calculation was done using the LRRb Reynolds stress equations in conjunction with the standard $\varepsilon$-equation 
Swirl Velocity with LRRb-RNG Model
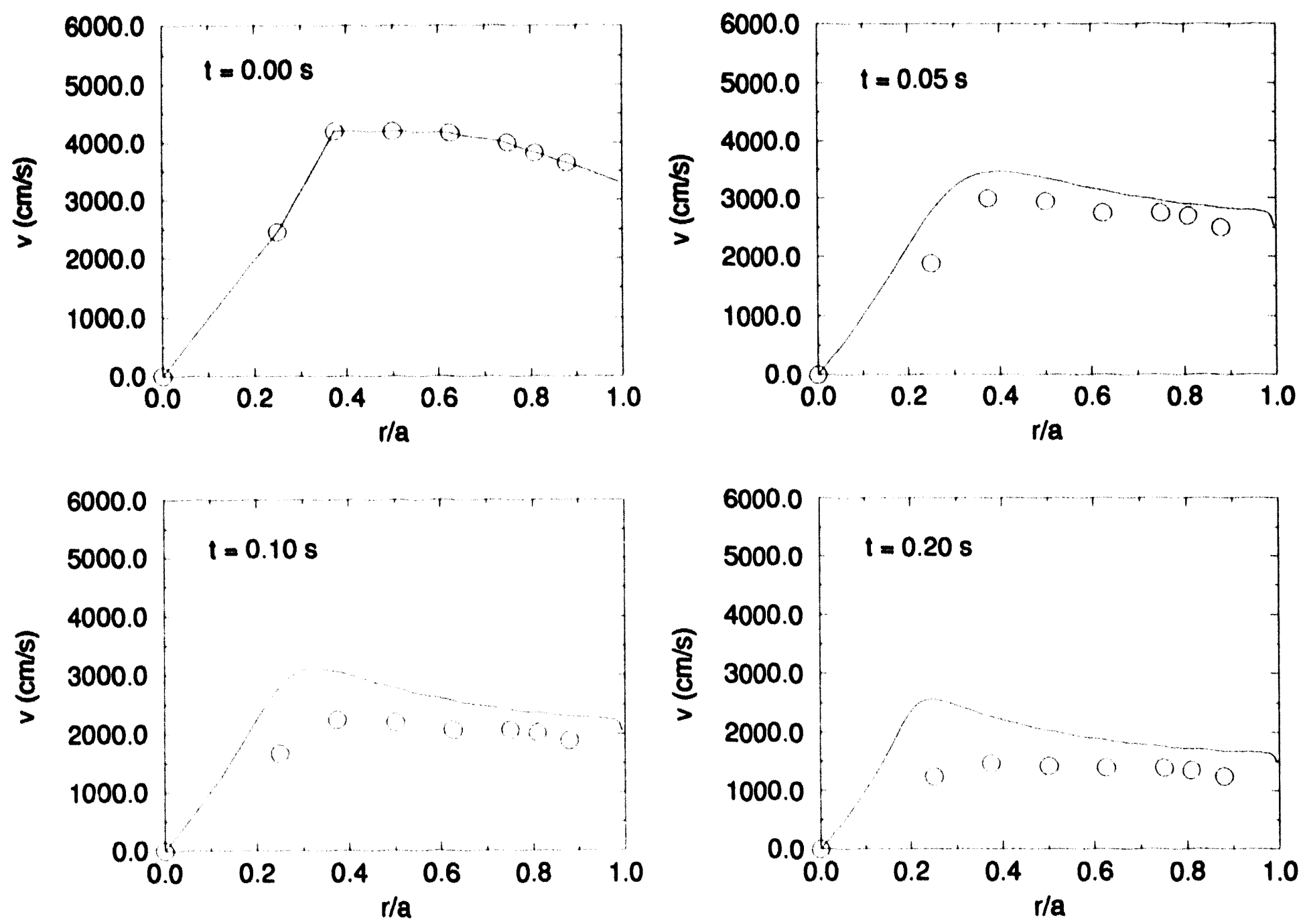

Figure 10: A time sequence of the azimuthal velocity profiles along the central plane as a function of radial position $r$ divided by the cylinder radius $a$. The solid lines are from a one-dimensional calculation and the circles correspond to experimental measurements. The calculation was done using the LRRb Reynolds stress equations in conjunction with the $\mathrm{RNG} \varepsilon$-equation. $\beta=0.04$. 
Türbulence Intensity with LRRb-RNG Mudel
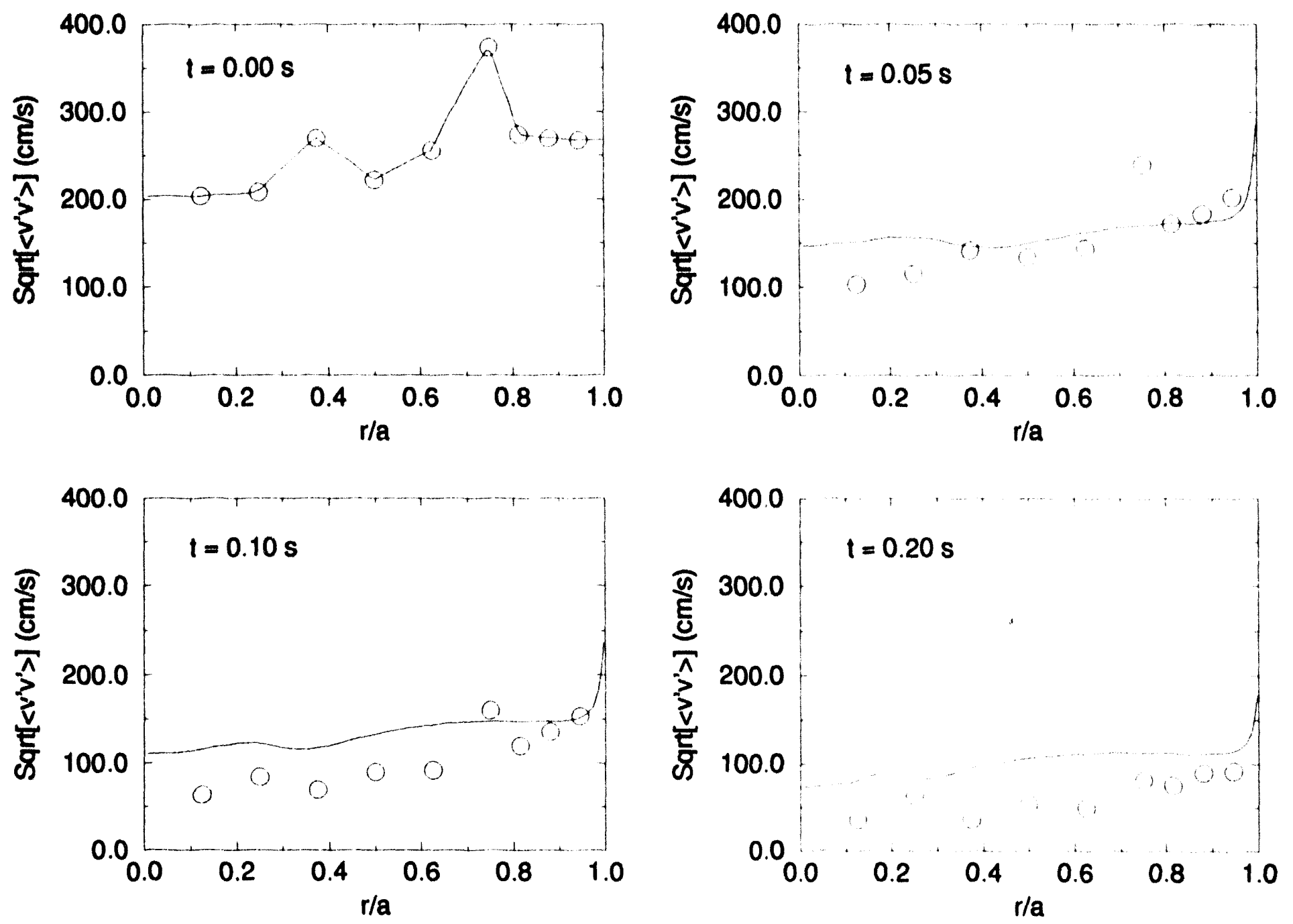

Figure 11: A time sequence of the turbulence intensity curves along the central plane as a function of radial position $r$ divided by the cylinder radius $a$. The turbulence intensity is the rms value of the fluctuating part of the azimuthal velocity. The solid lines are from a one-dimensional calculation, and the circles correspond to experimental measurements. The calculation was done using the LRRb Reynolds stress equations in conjunction with the RN(i E-equation. $\beta=0.04$. 
flow and turbulence intensity when the value of the additional model constant $\beta$ is suitably chosen.

Because of end wall effects, a two-dimensional calculation is necessary to quantitatively predict the mean velocity and the mean temperature field. Accordingly, we next plan to make an implicit implementation of the Reynolds stress evolution equations into a massively parallel, threedimensional hydrodynamics code. 


\section{References}

1. S. Wahiduzzaman and C. R. Ferguson, "Convective Heat Transfer from a Decaying Swirling Flow Within a Cylinder," Eighth International lleat Transfer Conference, Paper No. 86-IHTC-253, August (1986).

2. B.E. Launder, "Turbulence Modeling for the Nineties: Second Moment Closure ..... and Beyond ?," 12th International Conference on Numcrical Methods in Fluid Dymamics, K.W. Morton (ed.) (Springer-Verlag, New York, 1990 ) p.1.

3. V. Yakhot, S.A. Orszag, S. Thangam, T.B. Gatski, and ('. (i. Speziale, "I)evelopment of turbulence models for shear flows by a double expansion technique" Phys. Fluids A 4, 1510 (1992).

4. W.C. Reynolds, "Fundamentals of turbulence for turbulence modeling and simulation," Lecture Notes for Von Karman Institute, AGARI) Lecture Series No. 86, North Atlantic Treaty Organization (1987).

5. B.F. Schutz, Geometrical Methods of Mathematical Physics (Cambridge Iniversity Press, New York, 1980).

6. B.E. Launder, G. Reece, and W. Rodi, "Progress in the development of a Reynoldsstress turbulence closure," J. Fluid Mech. 68. 5337 (1975).

7. C.G. Speziale, S. Sarkar, and T.B. Gatski, "Modelling the pressure-st rain correlation of turbulence: an invariant dynamical systems approach," J. Fluid Mech. 227, 2 (1991).

8. J. Rotta, "Statistische Theorie Nichthomogener Turbulenz," Z. Phys. 129, 5.17 (1951).

9. S. Fu, B.E. Launder, and M.A. Leschziner, "Modelling Strongly Swirling Recirculating Jet Flow with Reynolds-Stress Transport Closures," Pror. Gth S'ymp. on Turbule mt Sher Flows, Paper 17.6, Toulouse, (1987).

10. L.D. Landau and E.M. Lifshitz, Fhud Mechanies, 2nd ed. (Pergamon Press. Vew York, 1987) p.172.

11. A.O. Demuren and S. Sarkar. "Systematic Study of Reynolds Stress ("losure Models in the ('omputations of Plane (hannel Flows," I( ASEL Report No. 92-19 (April 1992).

12. A.A. Amsden, P.J. O’Rourke, and T.D. Butler, "KIVA-II: A ('omputer Program for ('hemically Reactive Flows with Sprays," los Alamos National laboratory report IA. 11560)-MS (May 1989). 
Table 1: Table of Values of $b_{12}$ and 1/Pre $\mathrm{Pr}_{\varepsilon}$ Various Turbulence Models

\begin{tabular}{|l|c|l|}
\hline \hline \multicolumn{1}{|c|}{ Model } & $b_{12}$ & $1 / \mathrm{P}_{\boldsymbol{r}}$ \\
\hline Std $K-\varepsilon$ & -0.15 & 0.90 \\
LRRb & -0.13 & 0.59 \\
SSG & -0.16 & 1.09 \\
\hline
\end{tabular}

Table 2: Table of Values of $b_{12}$ and $\beta$ for Various Turbulence Models

\begin{tabular}{|l|c|r|}
\hline \hline \multicolumn{1}{|c|}{ Model } & \multicolumn{1}{c|}{$b_{12}$} & \multicolumn{1}{c|}{$\beta$} \\
\hline RNG $i-\varepsilon$ & -0.146 & 0.012 \\
LRRb & -0.128 & -0.007 \\
SSC & -0.160 & 0.060 \\
\hline
\end{tabular}



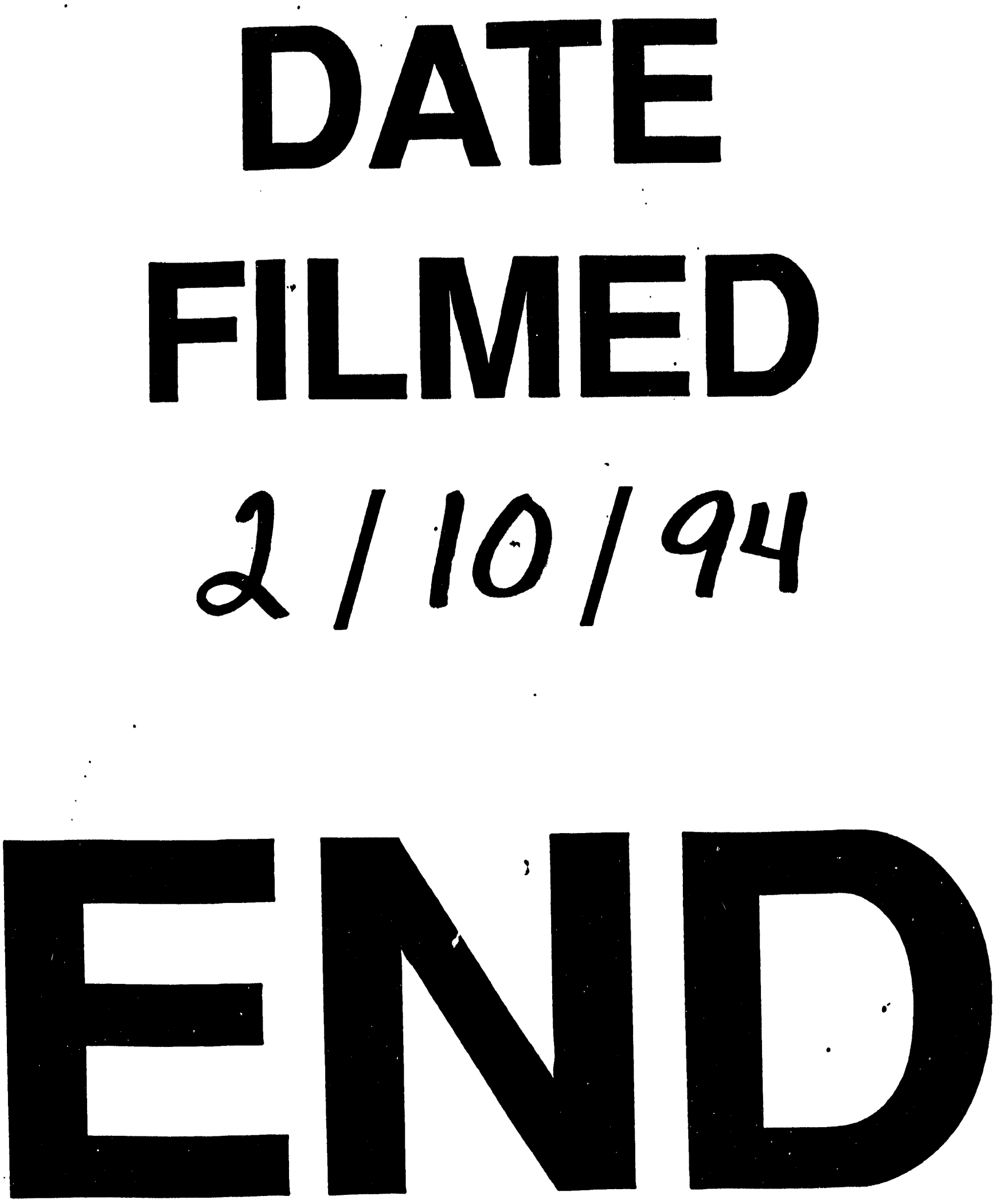
